\title{
Isolation and Characterization of Lytic Phage TUN1 Specific for Klebsiella Pneumoniae K64 Clinical Isolates From Tunisia
}

\section{Simone Eckstein}

Bundeswehr Institute of Microbiology, Munich Jana Stender

Bundeswehr Institute of Microbiology, Munich

\section{Sonia Mzoughi}

Department of Virology, Military Hospital of Instruction of Tunis

Kilian Vogele

Department of Physics, Technical University of Munich, Garching

\section{Jana Kühn}

Bundeswehr Institute of Microbiology, Munich

\section{Daniela Friese}

Bundeswehr Institute of Microbiology, Munich

\section{Christina Bugert}

Bundeswehr Institute of Microbiology, Munich

\section{Susann Handrick}

Bundeswehr Institute of Microbiology, Munich

\section{Mustapha Ferjani}

Department of Anesthesiology and Reanimation, Military Hospital of Instruction of Tunis

\section{Roman Wölfel}

Bundeswehr Institute of Microbiology, Munich

\section{Andrew Millard}

Department of Genetics and Genome Biology, University of Leicester

\section{Mohamed Ben Moussa}

Department of Virology, Military Hospital of Instruction of Tunis

Joachim J Bugert ( $\nabla$ joachim1bugert@bundeswehr.org)

Bundeswehr Institute of Microbiology, Munich

\section{Research Article}

Keywords: multidrug-resistant bacteria, Klebsiella pneumoniae, bacteriophage, phage therapy 
Posted Date: March 1st, 2021

DOl: https://doi.org/10.21203/rs.3.rs-199205/v1

License: (c) (i) This work is licensed under a Creative Commons Attribution 4.0 International License. Read Full License 


\section{Abstract \\ Background}

Multidrug-resistant Klebsiella pneumoniaespp $(K p)$ are emerging agents of severe infections of the respiratory and urinary tract or wounds, and progression to fatal septicemia. The use of bacteriophages is currently being considered as an effective alternative or adjuvant to antibiotic treatment.

\section{Results}

In this study, we report K-typing of 163 carbapenem-resistant Kp (CRKP) isolated 2014-2018 at the Military Hospital of Instruction of Tunis (MHT), Tunisia, using wzi gene sequencing. Thereby, we found that $\mathrm{K} 64 \mathrm{Kp}$ strains are associated with increased case/fatality rates, especially at the intensive care unit (ICU). Furthermore, we characterized a lytic $K p$ phage, vB_KpP_TUN1 (phage TUN1), isolated from waste water samples of the ICU at the MHT. Whole genome sequencing showed that this phage belongs to the Podoviridae family and encodes one putative depolymerase specifically digesting K64 Kp capsules. Furthermore, we could successfully assemble phage TUN1 in a non-replicative host (E. coli) raising the possibility of in vitro assembly in the absence of live bacterial hosts. Therefore, phage TUN1 is a promising candidate to be used as an adjuvant or an alternative to antibiotic therapy in CRKP infections, facilitating regulatory approval of phage therapy.

\section{Conclusions}

The lytic phage TUN1, efficiently lyses K64 Kp strains associated with increased case/fatality rates at body temperature. Together with its ability to be rescued in a non-replicative host these features enhance the utility of this phage as antibacterial agent.

\section{Introduction}

The spread of multidrug-resistant bacteria (MDRB) is an increasing global problem [1]. With reduced treatment options, the risk of life-threatening infections, including septicemia, grows and therefore new therapeutic approaches are required.

Bacteriophages targeting specific bacteria are considered a promising alternative to standard broadspectrum antibiotic therapy, especially in the case of MDRB infections.

The ESKAPEE group of bacterial pathogens comprising Enterococcus faecium, Staphylococcus aureus, Klebsiella pneumoniae, Acinetobacter baumannii, Pseudomonas aeruginosa, Enterobacter spp., and Escherichia coli [2] highlights the clinical impact of MDRB, and illustrates the prevalence of multidrugresistant gram-negative bacteria (MRGN) [3]. MRGN including Klebsiella pneumoniae $(K p)$ are also a problem in the military setting, indicated by the prevalence of MRGN in role 1 to 3 military treatment 
facilities [4]. Kp can be classified in four groups (MRGN 1 to 4) according to the level of antibiotic resistance. MRGN4 are resistant to carbapenem/ertapenem leaving colistin as only remaining treatment option [5]. The emergence of carbapenem-resistant $K p$ strains (CRKP) and their spread in hospitals is an alarming phenomenon reported to be correlated with high case/fatality rate of $40-70 \%[6,7]$. Therefore, CRKP are important nosocomial pathogens involved in serious and life-threatening infections, especially in the intensive care unit (ICU) [8] and of particular concern to clinicians [9]

$K p$ cells have typical polysaccharides capsules, which form biofilms, confer resistance to antibiotics, reduce phagocytosis, prevent a vigorous immune response and hence are a major $K p$ virulence factor [10]. Currently, more than $80 \mathrm{Kp}$ capsular types (K-types) are known [11]. The Wzi protein connects the polysaccharide capsule to the cell membrane of $K p$ [12]. Recently, Brisse et al. developed a PCR-based assay correlating a 580 bp sequence of genomic $K p$ wzi with high statistical significance to K-types [13].

As mentioned above, there is an urgent need for therapeutic approaches for CRKP infections alternative to antibiotic treatment. Recently, the use of bacteriophages was shown to be effective in the treatment of MDRB in a landmark clinical study [14]. Bacteriophages specific for $K p$ have been isolated previously [15-18], including phage specific for capsule type K24 [19]. Phages contain K-type-specific enzymes that depolymerize the polysaccharides. Thereby the phage can reach the host surface and thus bind to a secondary receptor like e.g. outer membrane proteins [20-22].

But, considering the wide variety of K-types, Klebsiella phages have to be isolated and characterized in large numbers to be used in therapeutic cocktails active against diverse $K p$ capsule types.

In this collaborative study between the Bundeswehr Institute of Microbiology (IMB) in Munich and the Military Hospital of Instruction of Tunis (MHT), we K-typed 168 CRKP obtained from a collection of strains, which were isolated from patients of different medical services at the MHT, Tunisia, between 2014 and 2018 [23] via wzi sequencing. Furthermore, we identified and characterized the lytic phage TUN1, a T7-type podovirus specific for $K p$ with the predominant capsule type K64 associated with increased case/fatality rates.

\section{Results}

The majority of $K p$ strains analyzed in this study $(70.2 \%)$ were isolated from patients in the MHT intensive care unit (ICU) followed by the unit for neonatology (10.7 \%). With $<3 \%$ each the remaining isolates were achieved from 13 other medical services at the MHT [23].

\section{The predominant capsule-type $\mathrm{K} 64$ is associated with increased case/fatality rates}

To determine the K-type for each $K p$ strain, wzi gene sequencing was performed as described by Brisse et al. 2013 [13]. 163 of $168 \mathrm{Kp}$ strains yielded the respective amplicon and 14 different K-types were found (Table 1). With 50.3 \% (82 CRKP strains), the most prevalent K-type was K64 followed by K17 abd K27 
( $23.9 \%$ and $9.8 \%$, respectively). For five $K p$ strains no amplification of the wzi gene locus could be achieved.

Table 1

K-genotype distribution of 163 clinical Kp strains

\begin{tabular}{|ll|}
\hline K-genotype & No. (\%) of strains \\
\hline K64 & $82(50.3)$ \\
\hline K17 & $39(23.9)$ \\
\hline K27 & $16(9.8)$ \\
\hline K52 & $6(3.7)$ \\
\hline K25 & $4(2.5)$ \\
\hline KN2 & $4(2.5)$ \\
\hline K55 & $4(2.5)$ \\
\hline K62 & $2(1.2)$ \\
\hline KL105 & $1(0.6)$ \\
\hline K2 & $1(0.6)$ \\
\hline K54 & $1(0.6)$ \\
\hline K3 & $1(0.6)$ \\
\hline K43 & $1(0.6)$ \\
\hline K58 & $1(0.6)$ \\
\hline
\end{tabular}

With 27 CRKP-related deaths, the overall case/fatality rate of CRPK infections at the MHT lies at $18.0 \%$. Unlike the second and third most Kp K-types (K17 and K27) K64 was associated with increased case/fatality in general but especially at the ICU (Fig. 1). With $63 \%$ and $70.3 \%$, respectively, almost two

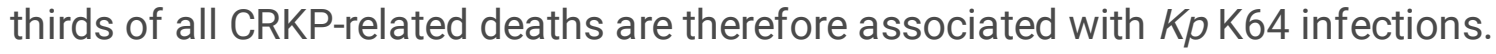

\section{Kp bacteriophage TUN1}

To isolate bacteriophages specific for the Tunisian CRKP strains wastewater was collected from locations at the MHT. For phage enrichment the $K p$ host strain 7984, exhibiting the most predominant capsule type K64, identified in this study, was used. However, plaque-forming phages could only be found in the wastewater collected from the ICU. One of them, vB_KpP_TUN1 (phage TUN1), was further characterized in this study. Initial experiments showed that this phage forms central clear plaques ( $\varnothing 1.5-$ $2 \mathrm{~mm}$ ) each surrounded by a halo $\left(\varnothing 5-6 \mathrm{~mm}\right.$ after $12 \mathrm{~h}$ at $37^{\circ} \mathrm{C}$ ) on $K p 7984$ (Fig. 2a). The growth curve showed that phage TUN1 has a replication cycle of $20 \mathrm{~min}$ and a burst size of 76 phage particles/cell (Fig. 2b). 
Analyzing the genome of phage TUN1 using high-throughput sequencing revealed a circular permuted genome consisting of 41,181 bp with a GC content of $52.9 \%$ and 53 predicted open reading frames (ORFs) as shown in Fig. 3 and Supplementary Table S1.

BlastN analysis found the most related genome sequence in the database to be Klebsiella phage 066037 (Accession No: MW042800.1, query coverage: $92 \%$ and $93.2 \%$ identity) belonging to the Przondovirus (Kp32virus) genus of the Podoviridae family, subfamily Autographiviridae in the order of Caudovirales [24].

Examining the phage's morphology using transmission electron microscopy (TEM), an icosahedral capsid with short tails could be observed (Fig. 4).

It has been described previously, that translucent halos surrounding phage plaques indicate the presence of phage-derived depolymerases [25]. Depolymerase activity of bacteriophages commonly lies within their tail fibers or tail spike proteins on the baseplate [26]. Nucleotide BLAST analysis revealed that the tail fiber protein, located downstream of internal virion protein D and upstream of class II holin (ORF 47; Fig. 3, Supplementary Table S1), exhibits the highest similarity (query coverage: $97 \%$; identity $96 \%$ ) with the tail fibers protein of Klebsiella phage P509. This phage also exhibits specific depolymerase activity against K64 Kp strains [27]. Therefore, the tail fiber protein is most likely to exhibit depolymerase activity.

\section{Phage TUN1 shows a narrow host range restricted to K64 Kp strains}

Since phage TUN1 was enriched on Kp 7984 a capsule type K64 strain, its specificity was investigated by testing 81 remaining Tunisian K64 Kp strains (Table 1) as well as representatives of other K-types as putative hosts. Eight representative strains are shown in Fig. 5. Phage TUN1 exhibits a narrow host range, as it was only able to lyse K64 Kp strains. Of $82 \mathrm{~K} 64 \mathrm{Kp}$ strains 57 were lysed by phage TUN1, 25 were not, including strain 6096 shown as example in Fig. 5. None of the non-K64 capsule types were lysed.

\section{Rescue of phage TUN1 in non-pathogenic E. coli cells}

For a potential therapeutic approach, genetic modification (e.g. to remove unwanted genetic elements like toxins, or genes allowing integration and a lysogenic state) and in vitro packing methods may be required, to facilitate regulatory approval. As a proof of principle that phage TUN1 can be rescued in a non-replicative host we transformed purified phage DNA into non-pathogenic E. coli NEBstable $\rightarrow 3040$ cells. Although no plaque formation on E. coli could be observed (not shown), phage TUN1 assembly within the cells was successful, proven by subsequent plaque assays with $K p$ hosts (Supplementary Figure S1).

\section{Discussion}


The increasing spread of MRGN is a global problem [1]. Especially carbapenem-resistant $K$. pneumoniae (CRKP) pose a high risk in hospitals and other medical facilities. Liangfei Xu et al., reported 2-fold increased mortality among CRKP infected patients compared to those infected with carbapenemsusceptible K. pneumoniae (CSKP) [7].

Thus, alternative therapeutic approaches to antibiotic treatment are required. For CRKP infections, capsule-specific lytic bacteriophages are promising candidates [14, 17-19]. Knowledge of the K-type of the respective $K$. pneumoniae strains is therefore imperative.

This study reports the use of a rapid method to predict the K-type of $K p$ first described by Brisse et al. [13]. In total, 163 CRKP strains from a previously described Tunisian clinical specimen collection [23] were capsule-typed. K-type K64 was not only identified as the most predominant K-type, but also as the K-type associated with the highest case/fatality rates of CRKP infected patients $(>70 \%$ in patients treated at the MHT ICU). This fits recent observations, both regarding the high case/fatality $[6,7]$ as well as the increased virulence of capsule type K64 [29].

However, the following limitations to this analysis have to be considered: $i$. the clinical specimen collection available lacks power in differentiating death caused by CRKP from any other factors, and it is difficult to draw definitive conclusions from current evidence because of the residual confounding factors and small sample sizes in many studies; ii. the study is retrospective in nature and thus susceptible to selection bias.

$K p$ can be found ubiquitously not only in human tissues but also in non-clinical sources, such as soil, drinking or surface waters and sewage [30]. This indicates that $K p$ phages should also be present in such environments and therefore easy to be isolated from nature.

In search of a potent tool to fight CRKP infections we looked for Klebsiella phages present at the MHT. Thereby we were able to obtain the lytic phage TUN1 from wastewater samples at the ICU using CRKP 7984 as a host strain. Based on BlastN analysis of the complete genome as well as morphologic characteristics phage TUN1 could be classified as bacteriophage of the genus Przondovirus, family Autographiviridae order Caudovirales, according to the newest species demarcation criteria set by the International Committee on Taxonomy of Viruses (ICTV) [24].

Phage TUN1 exhibited lysis specificity for Tunisian K64-type Kp strains. The replication cycle of 20 min and the burst size of 76 phage particles/cell are within the range described elsewhere [15-19]. The growth curve indicates that $\mathrm{MOI}=1$ reached approximately $60 \%$ of $K p$ cells, leading to a three-phased growth curve. While not classical, the three phases are distinguishable and similar enough to each other, to support the interpretation as a growth curve with 3 steps of 20 minutes, each, and a burst size of 76 particles per cell. As K64 was the most predominant K-type at the MHT, especially in the ICU, and all experiments were performed at $37^{\circ} \mathrm{C}$ representing human body temperature, phage TUN1 appears to be a good candidate for phage therapy. Furthermore, unlike e.g. the mycobacterium abscessus phage used for 
therapy by Dedrick et al. [14] TUN1 is a purely lytic phage and therefore does not need removal of lysogenic elements form the genome.

However, with $\sim 10$ minutes, the latent time of phage TUN1 was found to be relatively short and the burst size with 76 phage particles/cell was on the lower end of productivity. Furthermore, the putative depolymerase seems to be very specific for K64 Kp strains.

Additional restriction factors other than capsule type are most likely responsible for phage TUN1 being able to lyse more than two thirds but not all $K p$ strains of capsule type 64 .

For future phage therapy applications, all desirable features such as strong lytic activity, absence of virulence factors and high phage stability could be combined via genetic engineering. The use of genetic elements with depolymerase activity, targeting additional clinically relevant capsule types, may lead to phages with broader hostranges, and therefore more promising candidates for phage therapy based on phage TUN1.

Conducting initial proof-of-principle experiments, we were able to show that phage TUN1 can be rescued in a non-replicative host (E. coli). In vitro assembly of therapeutic bacteriophages in the absence of live bacterial hosts would simplify the regulatory approval as the most common factors of concern like the presence of endotoxins or the possibility of host-DNA contamination, would be absent by default. A collaboration with the Walter Reed Army Institute of Research (WRAIR) is aiming for the development of technical approaches to field deployable therapeutic phage cocktails [31-32].

The future goals of our current project at the Bundeswehr Institute of Microbiology are to i. identify and characterize bacteriophages against a broad range of $K p$ K-types found in German/Tunisian military and civilian hospitals; ii. develop technical approaches that allow genetic modification for the removal of unwanted genetic/lysogenic phage genome elements, possibly the addition of capsule specific factors and iii. work out a pipeline for therapeutic phage production, that implements the highest safety standards, facilitating regulatory approval.

Such a pipeline could then be applied in both civilian and military hospitals, and eventually extended to products able to withstand the harsher conditions in military field settings.

\section{Conclusion}

In conclusion, 163 carbapenem-resistant $K$. pneumonia strains from a previously published MHT collection were capsule-typed. Furthermore, a lytic phage, vB_KpP_TUN1, specific for Kp capsule type K64 was isolated from wastewater samples and analyzed for its host range, morphology and genomic characteristics. As K64 was found to be the most predominant K-type at the MHT, a potential application in the control of infections caused by multidrug-resistant $K$. pneumonia in Tunisia can be envisaged. Future studies will focus on improving the effectiveness and safety of $K p$-specific bacteriophages. 


\section{Materials And Methods}

\section{Clinical Kp strains used in this study}

168 clinical $K p$ strains were isolated and characterized as described elsewhere following the operating procedure of the Military Hospital of Tunis [23]. Isolates were recovered from anal swabs ( $n=35$ [20.83\%]), axillary swabs $(n=2[1.19 \%])$, a swab from a not-further defined location $(n=1[0.60 \%])$, materials from broncho-alveolar lavages $(n=6[3.57 \%])$, blood cultures $(n=53[31.54 \%])$, cytobacteriological examinations of sputum (CBES) $(n=5[2.97 \%])$, cerebrospinal fluid $(n=1[0.60 \%])$, exudates from the ear $(n=2[1.19 \%])$, biofilms at endotracheal tubes $(n=3[1.78 \%])$, gastric samples $(n=1$ $[0.60 \%])$, catheters $(K T)(n=19[11.30 \%])$, oral swabs $(n=1[0.60 \%])$, protected tracheal sampling materials (PTS) $(n=13[7.73 \%])$, pus $(n=18[10.71 \%])$, a medical device $(n=1[0.60 \%])$, and urine $(n=34$ [20.23\%]).

\section{Kp capsule typing}

To determine Kp capsular types (K-types), PCR-amplification of a 580-bp fragment of the wzi gene was performed according to Brisse et al., 2013 [13]. The respective PCR product was gel purified (QIAquick Gel Extraction Ki, Qiagen, Germany) and subsequently sent for sequencing (Eurofins Genomics, Germany).

wzi sequences were analyzed against the NCBI Genbank using BLASTN and the K-type alignments with the highest scores selected.

\section{Bacteriophage isolation}

Bacteriophages were isolated from wastewater samples from different locations in the MHT. To select bacteriophages, $10 \mathrm{~mL}$ of the sample was clarified by centrifugation at $7,000 \times \mathrm{g}$ for $10 \mathrm{~min}$ at $4^{\circ} \mathrm{C}$, followed by filtration of the supernatant through a $0.2 \mu \mathrm{m}$ pore size filter. $6.5 \mathrm{ml}$ of the water sample was then mixed with $6 \mathrm{ml} \mathrm{LB}+1 \mathrm{mM} \mathrm{CaCl}_{2}$ solution and $60 \mu \mathrm{l}$ of host bacterium using the strain 7984 (a K64type $K p$, the most common type found in the Tunis military hospital). For the host an over night $(\mathrm{O} / \mathrm{N})$ culture of the chosen host strain was diluted 1:100 in LB medium and grown until it reached an $0 D_{600}$ of $0.4-0.5$.

Subsequently, the mixture was incubated at $37^{\circ} \mathrm{C}$ for 42 hours. After $42 \mathrm{~h}$ the bacteria phage mixture was centrifuged at $7,000 \mathrm{xg}$ for $10 \mathrm{~min}$ at $4^{\circ} \mathrm{C}$ to spin down most bacteria. The supernatant was then filtrated through a $0.2 \mu \mathrm{m}$ pore size filter using a $20 \mathrm{ml}$ syringe into a sterile tube. The filtrate, containing possible phages, was stored at $4^{\circ} \mathrm{C}$ until further use.

\section{Plaque assay and plaque purification}

Plaque assays were conducted as described elsewhere [33]. In brief, $100 \mu$ filtrated phage-solution were mixed with $350 \mu \mathrm{l}$ of host $K p\left(\mathrm{OD}_{600} \mathrm{~nm}=0.4-0.6\right) .2 .5 \mathrm{ml}$ of $0.6 \% \mathrm{LB}-\operatorname{agar}\left(40^{\circ} \mathrm{C}\right)$ containing $1 \mathrm{mM} \mathrm{CaCl} \nabla$ were added and poured on a pre-prepared $2 \%(\mathrm{w} / \mathrm{v})$ LB-agar plate. When the toplayer has hardened the plates were inverted and incubated $\mathrm{O} / \mathrm{N}$ at $37^{\circ} \mathrm{C}$. 
To purify a phage plaque. $K p$ strains were grown in LB-broth containing $1 \mathrm{mM} \mathrm{CaCl}_{2}\left(37^{\circ} \mathrm{C}\right.$, shaking) to $\mathrm{OD}_{600}=0.4-0.6$. A single plaque from a previous plaque assay (based on size, reachability, representiveness) was picked with a sterile pipette tip, resuspended in a $15 \mathrm{~mL}$ Falcon with $350 \mu \mathrm{l}$ bacteria and filled up with LB-broth to $5 \mathrm{ml}$. The tube was incubated $\left(37^{\circ} \mathrm{C}\right.$, shaking) for $3-6$ hours. To remove the remaining bacteria the tube was centrifuged at $>7000 \mathrm{~g}$, for $10 \mathrm{~min}$, ate $4^{\circ} \mathrm{C}$. The supernatant was filtrated through a $0.2 \mu \mathrm{m}$ pore size filter under sterile conditions. The stock was stored at $4^{\circ} \mathrm{C}$ until further use.

\section{TEM}

Transmission electron microscopy was performed with a Philips CM 100 TEM using 2\% aqueous uranyl formate negative staining.

\section{Host-range determination}

Single colonies of $K p$ strains from a LB-agar-plate were resuspended in $5 \mathrm{~mL}$ LB-broth and $100 \mu$ leach were transferred into wells of a 96-well plate.

The phage stock was diluted to $10^{4} \mathrm{pfu} / \mathrm{ml}$ and $100 \mu \mathrm{l}$ of phage or LB-broth were added to the bacteria. Bacterial growth was then monitored by measuring the $\mathrm{OD}_{600 \mathrm{~nm}}$ every 60 minutes for 16 hours at $37^{\circ} \mathrm{C}$ under static conditions using a TECAN Spark Multireader.

\section{TUN1 growth curve}

Growth experiments were done as described by Delbrück and Ellis in 1939 [34]. Briefly, all $K p$ strains were grown in LB-broth containing $1 \mathrm{mM} \mathrm{CaCl}_{2}\left(37^{\circ} \mathrm{C}\right.$, shaking) to $\mathrm{OD}_{600} \mathrm{~nm}=0.4-0.6$. Klebsiella cells were counted in a haemocytometer and an infection with a $\mathrm{MOI}=1$ was prepared using a concentration of $1 \mathrm{x}$ $10^{4}$ in $10 \mathrm{~mL}$ LB-broth.

Every $10-30$ minutes a plaque assay with different dilutions $\left(10^{0}-10^{-2}\right)$ using the toplayer method with LB-agar $\left(0.6 \%, 40^{\circ} \mathrm{C}\right)$, containing $1 \mathrm{mM} \mathrm{CaCl}_{2}$, was performed. The plates were inverted and incubated overnight. On the next day titer and burst size were calculated by counting the plaque forming units (PFUs) using the formula: median number of plaques of $t_{\text {end }}$ of the replication cycle $/ t_{\text {start }}$ of the replication cycle for $\mathrm{MOI}=1$.

\section{Genome analysis}

The phage DNA was prepared with Lucigen MasterPure ${ }^{\mathrm{TM}}$ Complete DNA and RNA Purification Kit (Cat.No. MC85200). Approximately 1 ug of DNA was used in library preparation for minION sequencing using the ligation sequencing kit (SQK-LSK109) following the manufacturer`s instructions (Oxford Nanopore Technology, UK). Base-calling was carried out with guppy v3.4.3. Genome assembly was carried out with Unicycler v0.4.5 [35] using all reads $>5 \mathrm{~kb}$ in length, with further polishing using Medaka. The genome was initially annotated with Prokka, with further annotation using the RAST server [36], followed by 
manual curation. The complete sequence will be publicly available at Genbank (Acc.No: will be available upon publication)

Sequencing was conducted from DNA samples by the MWG Eurofins GATC sequencing service.

\section{Phage DNA transformation}

$5 \mu \mathrm{l}$ of purified phage DNA were added to E.coli NEBstable C3040 and transformed by heatshock $\left(42^{\circ} \mathrm{C}\right.$ for $90 \mathrm{sec}$ ). Subsequently, $1 \mathrm{ml}$ LB-broth was added and the bacteria were grown at $37^{\circ} \mathrm{C}$, shaking, for $2 \mathrm{~h}$. Half of the transformationproduct was then centrifuged and the pellet discarded. The transformationproduct was stored at $4^{\circ} \mathrm{C}$ and a plaque assay and a spot assay were performed on $\mathrm{Kp}$ strains.

\section{Declarations}

\section{Ethics approval and consent to participate:}

The clinical material used in this study was part of the retrospective and investigator-blinded study previously published by Kollenda et al. 2019 [23] for which ethical approval was waived by the Ethics Committee of the Medical Association of Hamburg, Germany (registration number WF-023/18). All procedures to obtain the bacterial isolate collection for this study were in accordance with the national Tunisian and German ethical standards and with the 1964 Helsinki declaration and its later amendments or comparable ethical standards. Informed consent for the study using the anonymized $K p$ isolate collection was waived as part of the vote of the Ethics Committee of the Medical Association of Hamburg, Germany (registration number WF-023/18).

The use of the $K p$ strains was authorized by the Tunisian coauthors of the study (S.M., M.F., and M.B.M.) in a MTA between IMB and MHT.

\section{Consent for publication}

Not applicable.

\section{Availability of data and materials}

The phage genome datasets generated and analysed during the current study are available at the European Nucleotide Archive (ENA) - https://www.ebi.ac.uk/ena/browser/view/PRJEB37291; sequence ID ERZ1741895.

\section{Competing interests}

The authors declare that they have no competing interests.

\section{Author Contributions:}


Conceptualization: J.J.B.

Methodology: J.J.B., S.M., J.S, K.V., J.K., D.F., C.B., and A.M.

Software S.E. and A.M.

Validation: S.E., J.S., and J.J.B.

Formal analysis, S.E., J.S., S.M., A.M., and J.J.B.

Investigation: J.J.B.

Resources: J.J.B., S.H., A.M., and R.W.

Data curation: J.S., A.M. and D.F

Writing-original draft preparation: S.E., J.S., S.M., and J.J.B.

Writing-review and editing: S.E., J.S. A.M. and J.J.B.

Visualization: S.E., J.S. and J.J.B.

Supervision in hospital and lab: J.J.B. (IMB), M.F. and M.B.M. (MHT)

Project administration: J.J.B.

Funding acquisition: R.W., S.H. and J.J.B.

All authors have read and agreed to the published version of the manuscript.

\section{Funding:}

This study was funded by the Medical Biological Defense Research Program of the Bundeswehr Medical Service.

\section{Acknowledgments:}

The authors thank Dr. Brett E. Swierczewski, CIDR/WRAIR, Silverspring, MD, U.S.A., Professor Christian Willy, Bundeswehrkrankenhaus Berlin, Germany, and members of the Bundeswehr Institute of Microbiology for critical reading of the manuscript.

Conflicts of Interest: The authors declare no conflict of interest.

\section{References}


1. WHO: Antimicrobial resistance https://www.who.int/news-room/fact-sheets/detail/antimicrobialresistance [Accesed: 18.12.2020]

2. Mulani, M.S.; Kamble, E.E.; Kumkar, S.N.; Tawre, M.S. and Pardesi, K.R. Emerging Strategies to Combat ESKAPE Pathogens in the Era of Antimicrobial Resistance: A Review. 2019; Front Microbiol. 10: 539. doi: 10.3389/fmicb.2019.00539

3. Kaase, M. MRGN: neue Klassifikation für multiresistente gramnegative Bakterien. 2013. J Lab Med, 37(6), 299-304. doi: https://doi.org/10.1515/labmed-2012-0067

4. Lesho EP, Clifford RJ, Chukwuma U, Kwak YI, Maneval M, Neumann C, Xie S, Nielsen LE, Julius MD, McGann P, Waterman PE. Carbapenem-resistant Enterobacteriaceae and the correlation between carbapenem and fluoroquinolone usage and resistance in the US military health system. 2015Diagn Microbiol Infect Dis. 81(2):119-25. doi: 10.1016/j.diagmicrobio.2014.09.017. Epub 2014 Sep 28. PMID: 25497458.

5. Marchaim, D.; Navon-Venezia, S.; Schwaber, M.J.; Carmeli, Y. Isolation of imipenem-resistant Enterobacter species: emergence of KPC-2 carbapenemase, molecular characterization, epidemiology, and outcomes. 2008 Antimicrob Agents Chemother, 52:1413-1418.

6. Decré, D.; Verdet, C.; Emirian, A.; Le Gourrierec, T.; Petit, J.C.; Offenstadt, G.; Maury, E.; Brisse, S.; Arlet, G. Emerging severe and fatal infections due to Klebsiella pneumoniaein two university hospitals in France. 2011 Clin. Microbiol. 49:3012-3014

7. Liangfei, X., Xiaoxi, S.; Xiaoling, M. Systematic review and meta-analysis of mortality of patients infected with carbapenem-resistant Klebsiella pneumonia. 2017Ann Clin Microbiol Antimicrob. 16(1):18. doi: 10.1186/s12941-017-0191-3.

8. Gasink, L.B.; Edelstein, P.H.; Lautenbach, E.; Synnestvedt, M.; Fishman, N.O. Risk factors and clinical impact of Klebsiella pneumoniae carbapenemase- producing pneumoniae. 2009 Infect Control Hosp Epidemiol; 30:1180- 1185.

9. Pitout, J.D.D.; Laupland, K.B. Extended-spectrum $\beta$-lactamase-producing Enterobacteriaceae: an emerging public-health concern. 2008 Lancet Infect Dis.;8(3):159-166

10. Paczova, M.K.; Mecsas, J. Klebsiella pneumoniae: going on the offense with a strong defense. 2016 Microbiol Mol Biol Rev, 80:629-61

11. Pan, Y.J.; Lin, T.L.; Chen, C.T.; Chen, Y.Y.; Hsieh, P.F.; Hsu, C.R.; Wu, M.C.; Wang, J.T. Genetic analysis of capsular polysaccharide synthesis gene clusters in 79 capsular types of Klebsiella2015Sci. Rep., 5, 1-10. DOI: 10.1038/srep15573.

12. Bushell, S.R.; Mainprize, I.L.; Wear, M.A.A.; Lou, H., Whitfield, C. and Naismith, J.H. Wzi Is an Outer Membrane Lectin that Underpins Group 1 Capsule Assembly in Escherichia coli. 2013 Structure. 7; 21(5): 844-853.

13. Brisse, S.; Passet, V.; Björk Haugaard, A.; Babosan, A.; Kassis-Chikhani, N.; Struve, C.; Decré, D. wzi Gene Sequenzing, a Rapid Method for Determination of Capsular Type for Klebsiella2013 JCM

14. Dedrick, R.M., Guerrero-Bustamante, C.A., Garlena, R.A. et al. Engineered bacteriophages for treatment of a patient with a disseminated drug-resistant Mycobacterium abscessus. 2019 Nat Med 25, 730- 
733 doi:10.1038/s41591-019-0437-z

15. Pan, Y.J.; Lin, T.L.; Chen, Y.Y.; Lai, P.H.; Tsai, Y.T.; Hsu, C.R.; Hsieh, P.F.; Lin, Y.T.; Wang, J.T. Identification of three podoviruses infecting Klebsiella encoding capsule depolymerases that digest specific capsular types. 2019Microb Biotechnol. May;12(3):472-486. doi: 10.1111/1751-7915.13370.

16. Kęsik-Szeloch, A., Z. Drulis-Kawa, B. Weber-Dąbrowska, J. Kassner, G. Majkowska-Skrobek, D. Augustyniak, M. Łusiak-Szelachowska, M. Żaczek, A. Górski and A. M. Kropinski. Characterising the biology of novel lytic bacteriophages infecting multidrug resistant Klebsiella pneumoniae. 2013 Virology Journal 10: 100-100.

17. Townsend, E., Kelly, L., Gannon, L., Muscatt, G., Dunstan, R., Michniewski, S., Sapkota, H., Kiljunen, S.J., Kolsi, A., Skurnik, M., Lithgow, T., Millard, A.D., Jameson, E. Isolation and characterisation of Klebsiellaphages for phage therapy. 2020 bioRxiv https://doi.org/10.1101/2020.07.05.179689

18. Anand, T., Virmani, N., Kumar, S., Mohanty, A.K., Pavulraj, S., Bera, B.C., Vaid, R.K., Ahlawat, U., Tripathi, B.N. Phage therapy for treatment of virulent Klebsiella pneumoniae infection in a mouse model. 2020 J Glob Antimicrob Resist. 21:34-41. doi: 10.1016/j.jgar.2019.09.018.

19. Horváth M, Kovács T, Koderivalappil S, Ábrahám H, Rákhely G, Schneider G. Identification of a newly isolated lytic bacteriophage against K24 capsular type, carbapenem resistant Klebsiella pneumoniae2020Sci Rep.;10(1):5891. doi: 10.1038/s41598-020-62691-8. PMID: 32246126; PMCID: PMC7125228.

20. Zhao X, Cui Y, Yan Y, Du Z, Tan Y, Yang H, Bi Y, Zhang P,Zhou L, Zhou D, Han Y, Song Y, Wang X, Yang R Outermembrane proteins ail and OmpF of Yersinia pestis are involvedin the adsorption of T7related bacteriophage Yep-phi. 2013 J Viro/ 87(22):12260-12269. https ://doi.org/10.1128/JVI.01948-13

21. Fan F, Li X, Pang B, Zhang C, Li Z, Zhang L, Li J, Zhang J, Yan M, Liang W, Kan B The outermembrane protein TolC of Vibrio cholerae serves as a second cell-surface receptor for the VP3 phage 2018. J Biol Chem 293(11):4000-4013. https ://doi.org/10.1074/jbc.M117.80568 9

22. Porcek, N.B.; Parent K.N. Key residues of S flexneri OmpA mediate infection by bacteriophage Sf6. 2015 J Mol Biol 427(10):194-196. https://doi.org/10.1016/j.jmb.2015.03.012

23. Kollenda et al., Screening for carbapenemases in ertapenem-resistant Enterobacteriaceae collected at a Tunisian hospital between 2014 and 2018. European Journal of Microbiology and Immunology 9(2019)1, pp. 9-13

24. Schoch, C. L., Ciufo, S., Domrachev, M., Hotton, C. L., Kannan, S., Khovanskaya, R., Leipe, D., Mcveigh, R., O'Neill, K., Robbertse, B., Sharma, S., Soussov, V., Sullivan, J. P., Sun, L., Turner, S., \& KarschMizrachi, I. NCBI Taxonomy: a comprehensive update on curation, resources and tools. 2020 Database: the journal of biological databases and curation. https://doi.org/10.1093/database/baaa062.

25. Lin, T.L., Hsieh, P.F., Huang, Y.T., Lee, W.C., Tsai, Y.T., Su, P.A., et al. Isolation of a bacteriophage and its depolymerase specific for K1 capsule of Klebsiella pneumoniae: implication in typing and treatment. 
$2014 \mathrm{~J}$ Infect Dis 210: 1734-1744.

26. Latka, A.; Maciejewska, B.;Majkowska-Skrobek, G.; Briers, Y.;Drulis-Kawa, Z. Bacteriophage-encoded virion-associated enzymes to overcome the carbohydrate barriers during the infection process App/ Microbiol Biotechno/2017, 101:3103-3119

27. Li, M., Xiao, Y., Li, P. et al. Characterization and genome analysis of Klebsiella phage P509, with lytic activity against clinical carbapenem-resistant Klebsiella pneumoniae of the KL64 capsular type. 2020 Arch Virol 165, 2799-2806. https://doi.org/10.1007/s00705-020-04822-0

28. Dedrick, R.M., Guerrero-Bustamante, C.A., Garlena, R.A. et al. Engineered bacteriophages for treatment of a patient with a disseminated drug-resistant Mycobacterium abscessus. 2019 Nat Med 25, 730733 doi:10.1038/s41591-019-0437-z

29. Yang, Q., Jia, X., Zhou, M., Zhang, H., Yang, W., Kudinha, T., Xu, Y..Emergence of ST11-K47 and ST11K64 hypervirulent carbapenem-resistant Klebsiella pneumoniaein bacterial liver abscesses from China: a molecular, biological, and epidemiological study. 2020 Emerg Microbes Infect. 9, 320-331. doi: 10.1080/22221751.2020.1721334.

30. Bagley ST. Habitat association of Klebsiella1985/nfect Control. 6(2):52-8. doi: 10.1017/s0195941700062603. PMID: 3882590.

31. NATO STANDARD AJP-4.10 ALLIED JOINT DOCTRINE FOR MEDICAL SUPPORT - WRAIR - phage group (Brett E. Swierczewski, Bacterial Diseases Branch (BDB), Center for Infectious Diseases Research (CIDR),Walter Reed Army Institute of Research (WRAIR): The US Army Bacteriophage Therapeutics Program for the Treatment of Multidrug Resistant Wound Infections WMM 2020 64(6-7)

32. Hung, C.-H., C.-F. Kuo, C.-H. Wang, C.-M. Wu and N. Tsao. Experimental Phage Therapy in Treating Klebsiella pneumonia Mediated Liver Abscesses and Bacteremia in Mice. 2011Antimicrob Agents Chemother 55(4): 1358

33. Delbrück and Ellis (1939) - Figure from Prescott Harley Klein's Microbiology, 7th edition, p430

34. Wick RR, Judd LM, Gorrie CL, Holt KE. Unicycler: resolving bacterial genome assemblies from short and long sequencing reads.2017PLoS Comput Biol.

35. Aziz RK, Bartels D, Best AA, DeJongh M, Disz T, Edwards RA, Formsma K, Gerdes S, Glass EM, Kubal $M$, Meyer F, Olsen GJ, Olson R, Osterman AL, Overbeek RA, McNeil LK, Paarmann D, Paczian T, Parrello B, Pusch GD, Reich C, Stevens R, Vassieva O.; Vonstein, V.; Wilke, A.; Zagnitko, O. The RAST server: rapid annotations using subsystems technology. 2008BMC Genomics 9:75.

\section{Figures}




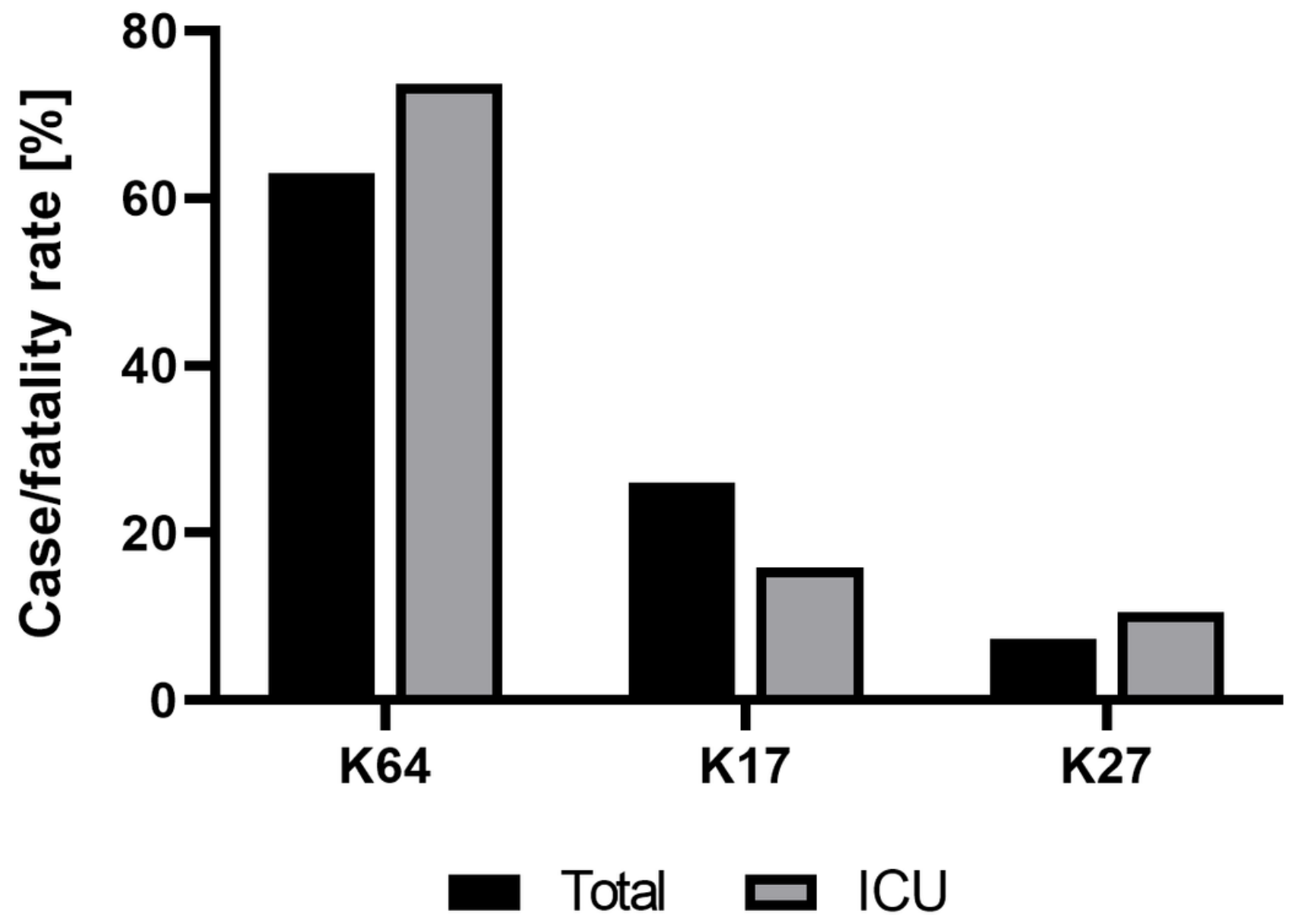

Figure 1

Case/fatality rates associated with Kp K-types K64, K17 and K27. Overall case/fatality rates (black) and case/fatality rates at the ICU (grey) in relation to CRKP-related deaths caused by Kp K64, K17 or K27 strains. 

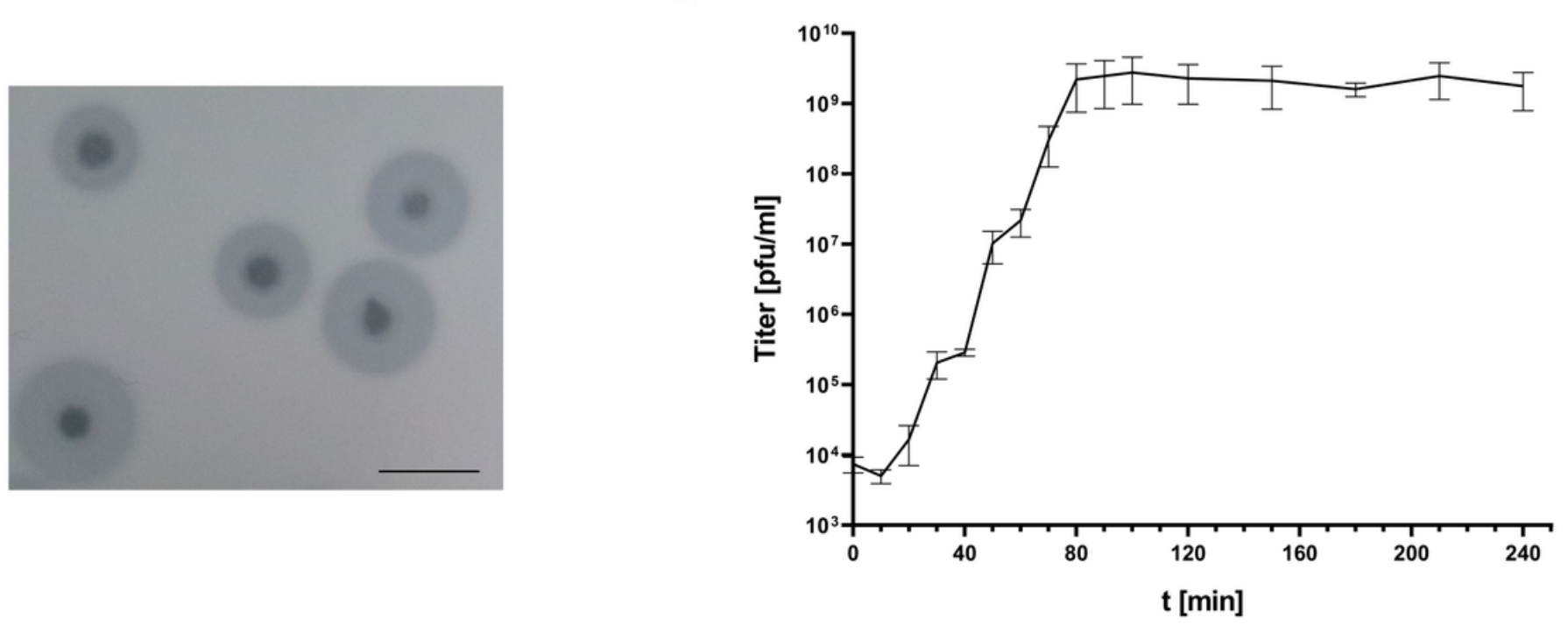

Figure 2

Initial characterization of phage TUN1 with its host Kp 7984. (a) Plaque assay revealing clear plaques each surrounded by a translucent halo. Scale bar: $50 \mathrm{~mm}$ (b) Growth curve of phage TUN1 was carried out at $\mathrm{MOI}=1$. Data is shown as mean from three biological experiments. Error bars represent standard deviation.

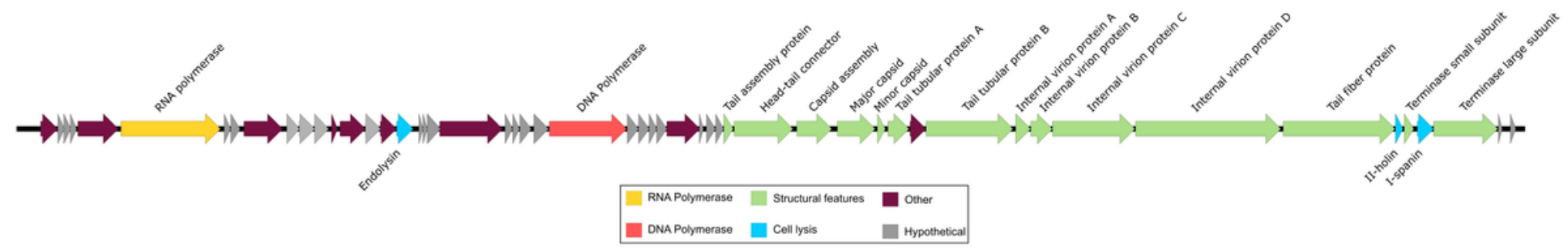

Figure 3

Schematic overview of the genome of phage TUN1. ORF coding for RNA polymerase is shown in yellow, while the ORF encoding a DNA polymerase is shown in red. ORFs coding for proteins involved in phage particle assembly and packaging are marked in green, and ORFs coding for enzymes involved in host lysis are marked in blue. Other functional ORFs are marked in purple while ORFs of hypothetical proteins are marked in grey. Arrows indicate the direction of transcription. 


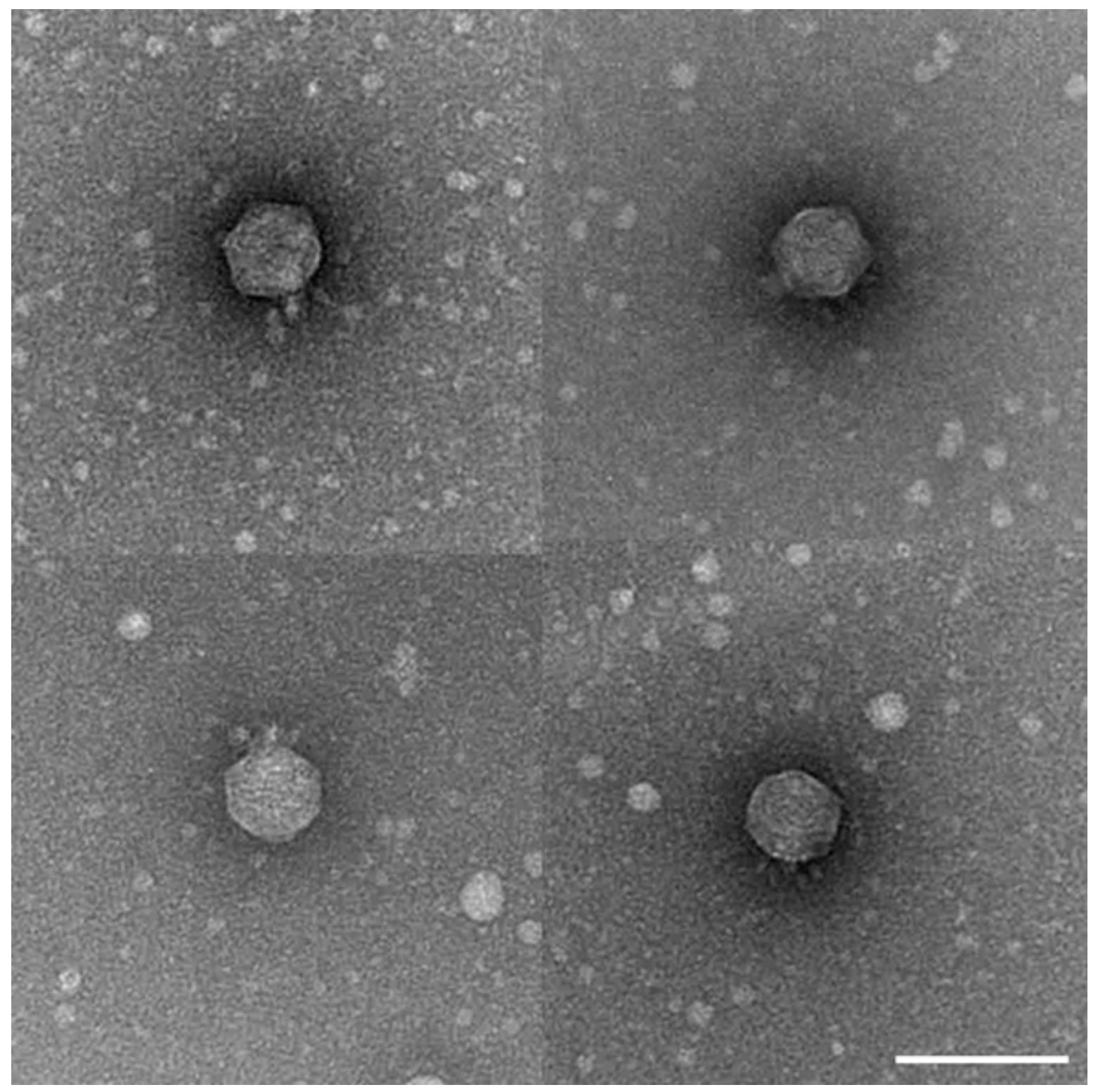

\section{Figure 4}

TEM of phage TUN1. Four TEM images showing one TUN1 phage particle each reveal the characteristic morphology of podoviruses. Scale bar: $100 \mathrm{~nm}$ 

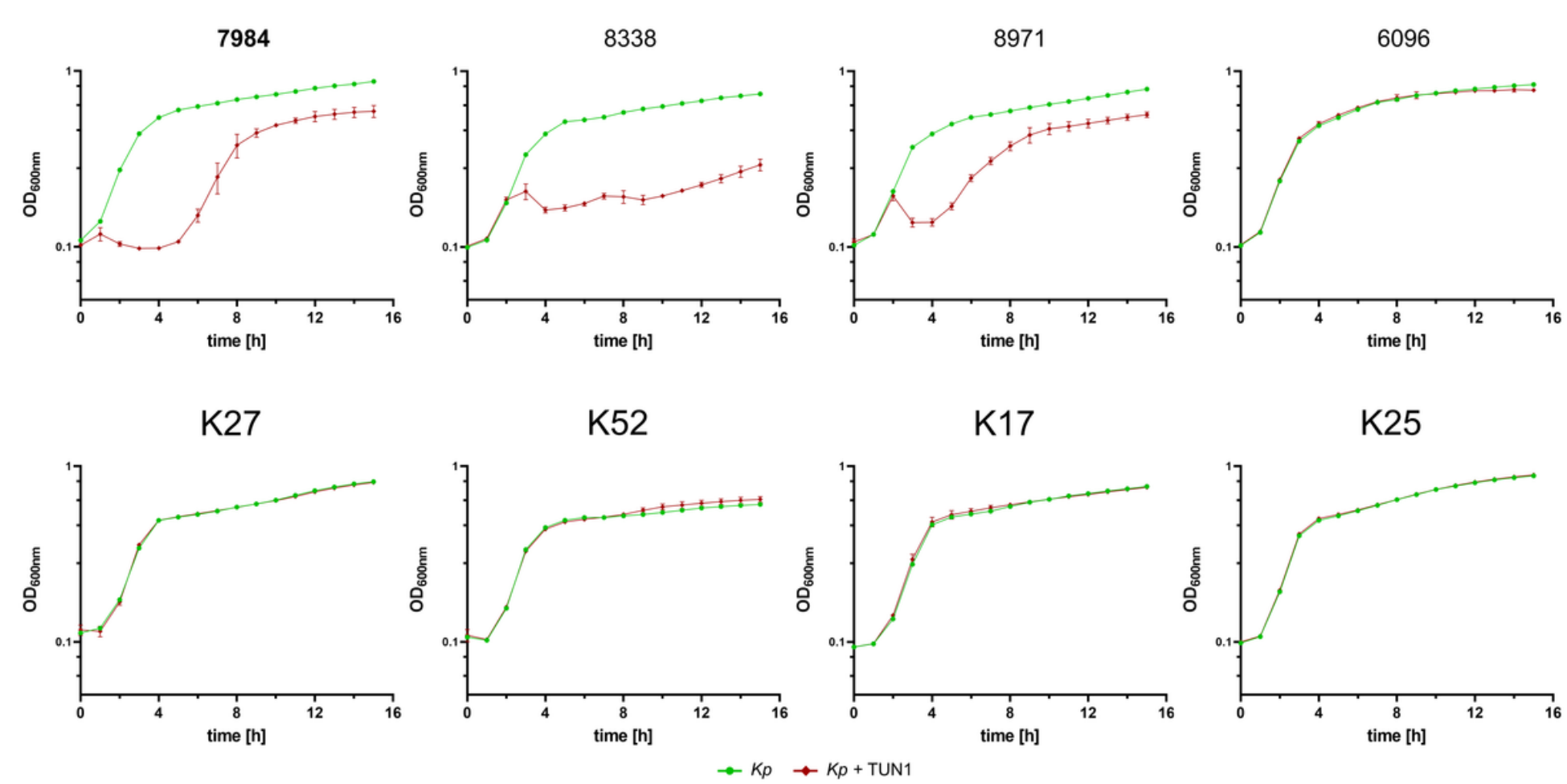

Figure 5

Lytic activity of phage TUN1 against Kp strains with different K-types. Upper panel: Growth experiments with phage TUN1 and four different Kp K64 strains. TUN1 lyses its host strain used for enrichment: Kp 7984 (written in bold) as well as Kp 8338 and 8971 with different efficiencies while Kp 6096 is not affected. Lower panel: Effect of phage TUN1 on Kp with the capsule types K27, K52, K17 and K25. Here, no lysis of Kp by phage TUN1 could be observed. Green: Kp strain alone. Red: Kp strain supplemented with phage TUN1

\section{Supplementary Files}

This is a list of supplementary files associated with this preprint. Click to download.

- EcksteinetalBMCMicrobiologySupplementaryMaterials.pdf 